\title{
Homomorphic Encryption-enabled Distance-based Distributed Formation Control with Distance Mismatch Estimators
}

\author{
Mariano Perez Chaher ${ }^{1}$, Bayu Jayawardhana ${ }^{1}$, and Junsoo Kim ${ }^{2}$
}

\begin{abstract}
This paper considers the use of homomorphic encryption for the realisation of distributed formation control of multi-agent systems via edge computer. In our proposed framework, the distributed control computation in the edge computer uses only the encrypted data without the need for a reset mechanism that is commonly required to avoid error accumulation. Simulation results show that, despite the use of encrypted data on the controller and errors introduced by the quantization process prior to the encryption, the formation is able to converge to the desired shape. The proposed architecture offers insight on the mechanism for realising distributed control computation in an edge/cloud computer while preserving the privacy of local information coming from each agent.

Index Terms-Distributed formation control, Homomorphic encryption
\end{abstract}

\section{INTRODUCTION}

Encryption standards such as the Advanced Encryption Standard (AES) have provided a reliable method to ensure data remains private [1]. However, the development of network computing and the Internet of Things, that is, the collaboration of different nodes within a network, have brought up concerns on the privacy and security of data that require new solutions [2]. The introduction of $5 \mathrm{G}$ communication technology and beyond, which guarantees ultra low-latency and high reliability of the wireless network, paves way for the implementation of cloud-based or edge-based control systems to control complex systems over a wide geographical area with minimal infrastructure footprint. While real-time data can reliably be transferred over air without wired/cabled connections, the issue of privacy and safety of critical realtime data transmission has been a major concern in the adoption of $5 \mathrm{G}$ wireless network by the high-tech industry.

Data sharing when working with different nodes in a network is inevitable. The security compromise is created by the availability of plaintext data in an untrustworthy third-party managed node that can be directly accessed by malicious agents. This problem manifests itself in Networked Control Systems (NCS) [3] when cloud-based or edgebased control systems are deployed. In order to perform the operations needed, an NCS using current encryption standards requires data to be decrypted at each node. The distribution of plaintext data across the network can be avoided by using Homomorphic Encryption (HE). By allowing for the manipulation of data in its ciphertext form it provides

\footnotetext{
${ }^{1}$ M. Perez Chaher and B. Jayawardhana are with the Engineering and Technology Institute Groningen, Faculty of Science and Engineering, University of Groningen, 9747AG Groningen, The Netherlands.

${ }^{2}$ J. Kim is with the Division of Decision and Control System, KTH Royal Institute of Technology, Sweden.
}

another layer of security [4] which has been used in NCS to guarantee the privacy of real-time data of sensor and control signals $[5]-[8]$.

Although HE provides further security to network computing by allowing for addition and multiplication of ciphertexts, it also has its own drawbacks depending on the chosen encryption algorithm. For HE schemes based on the Learning With Errors (LWE) problem, the limitations are the gradual corruption of data depending on the amount of ciphertext operations, and the number of operations other than addition and multiplication, in case bootstrapping techniques are not utilized for the sake of real-time operation [6]. These issues are especially challenging for dynamic controllers which require recursive updates of the system's state. Two methods exist to mitigate this limitation.

An approach proposed by Murguia et al. [9] is to periodically reset the controller's state to its initial value. Alternatively, bootstrapping can be used to refresh the state unlimitedly [10]. These methods increase computational complexity of the process or lead to performance degradation, making them less attractive for practical uses. In this work, we consider integral controller design so that, following the observations of previous research [11], [12], it is made possible to update the encrypted system's state for an infinite time horizon without its reset, nor performance degradation. In particular, we present a scheme that allows for the control of a distributed dynamic multi-agent formation using homomorphic encryption.

Distributed formation control refers to the design of distributed controllers for steering all robots to reach and maintain a prescribed formation shape based on local information from on-board sensor systems. The desired formation shape is typically defined using the specific relative information to the neighbours, such as, distance, relative position, feature or bearing information. Correspondingly, the local controller uses the same information obtained from the sensor to steer the robot towards the right direction and distance. We refer to [13] for a comprehensive review on the gradientbased formation control approaches. Among these methods, distance-based approach has been widely adopted as it is based on local coordinate frame, as well as, local relative measurement, enabling full distributed implementation of the control law [14]. Once the formation shape can be maintained, high-level group tasks can be carried out accordingly, such as, performing group motion [15].

While a distance-based formation control law can be implemented locally, it can induce undesired formation shapes and group motion when there are mismatches in the distance 
constraints or measurement biases between pairing agents [16]. In order to tackle this unwanted behaviour, dynamic estimators can be deployed which compensate these mismatches or biases [14].

In this paper, we implement and evaluate the use of HEenabled distance-based formation control law with mismatch estimators via edge/cloud computer. The control computation for each agent is performed independently of that of the other agents; hence it is "locally" computed in the edge/cloud. This allows us to offload computational resources from the robotic agent to the edge/cloud and enable complex tasks computation (such as, SLAM and collision avoidance) to be done in the same local environment in the edge/cloud. In practice, the real-time transmission of the sensor data and control input data will be enabled by means of low-latency wireless network of $5 \mathrm{G}$ or beyond. The use of infinite-time horizon $\mathrm{HE}$ in this context is to secure the privacy of both the sensor data and control data in the edge/cloud while enabling us to achieve the desired formation shape. We propose the use of scaled logarithmic quantizer prior to the encryption for maintaining precision and investigate the role of encryption key length to the overall performance. In our simulation, we show that the formation performance of the multi-agent systems does not deteriorate in spite of the presence of mismatches.

The paper is organized as follows. Section II presents the preliminaries on the encryption process and the distributed formation control with estimators. In Section III, we present the use of scaled logarithmic quantizer and sets out the HE-enabled distributed formation control architecture. We present the simulation setup and results in Section IV. The conclusions and future works are presented in Section $\mathrm{V}$

\section{HOMOMORPHIC ENCRYPTION AND DISTANCE-BASED DISTRIBUTED FORMATION CONTROL WITH ESTIMATOR}

In this section, we will provide some preliminaries that covers the two main topics on $\mathrm{HE}$ and on robust distributed formation control which employs distributed state estimators to compensate for distance mismatches and measurement biases. In the first two subsections, we present $\mathrm{HE}$ for standard feedback control systems [6] and a method to ensure the encryption in the closed-loop systems is operational for an infinite time horizon without the need to reset the states [11]. In the following two subsections, we introduce the distributed formation control methods [15] with its associated dynamic estimator that compensates for the measurement bias.

\section{A. Homomorphic Encryption}

Let us revisit HE that allows for both the addition and multiplication operations to be done in ciphertexts. Firstly, we need to define some notation. With a positive integer $p \in \mathbb{N}$, which is chosen as a power of 10 for convenience, we define the space of plaintexts, as $\mathbb{Z}_{p}:=\left\{m \in \mathbb{Z}:-\frac{p}{2} \leq\right.$ $\left.m<\frac{p}{2}\right\}$, whose cardinality is $p$, so that an element $m \in \mathbb{Z}_{p}$ represents a message in its plaintext form. Let $L$ be also a power of 10 and $q=L p$, so that the space $\mathbb{Z}_{q}$ of ciphertexts is defined as $\mathbb{Z}_{q}:=\left\{m \in \mathbb{Z}:-\frac{q}{2} \leq m<\frac{q}{2}\right\}$. Finally, a secret key used to decrypt and encrypt a message $m$ is denoted by $s k$, which is an integer vector of size $N$ such that $s k \in \mathbb{Z}_{q}^{N}$.

When a message $m \in \mathbb{Z}^{n \times 1}$ is encrypted, two new random matrices are generated: $A \in \mathbb{Z}_{q}^{n \times N}$, and injected error $e \in$ $\mathbb{Z}^{n}$ whose elements are sampled from zero-mean discrete Gaussian distribution 1 , where $n$ is the dimension of $m$. Using these components, the message $m$ is encrypted as follows

$$
\operatorname{Enc}(m)=[(-A \cdot s k+L m+e) \bmod q, A]=: \mathbf{m},
$$

where mod is the (component-wise) modulo operation defined as $a \bmod q:=a-\lfloor(a+(q / 2)) / q\rfloor q$ for $a \in \mathbb{Z}$ and $q \in \mathbb{N}, \mathbf{m} \in \mathbb{Z}_{q}^{n \times(N+1)}$ is the encrypted form of $m,[\cdot, \cdot]$ denotes the concatenation of two matrices, and $\lfloor\cdot\rfloor$ the floor function.

Subsequently, the decryption process can be done by using a secret key vector $s:=\left[1, s k^{T}\right]^{T}$. Hence, the ciphertext $\mathbf{m}$ is decrypted as

$$
\operatorname{Dec}(\mathbf{m})=\left\lceil\frac{(\mathbf{m} \cdot s) \bmod q}{L}\right\rfloor=\left\lceil\frac{L m+e}{L}\right\rfloor=: m,
$$

where $\lceil\cdot\rfloor$ is the element-wise rounding operation.

These particular encryption and decryption processes allow us to use homomorphic operations between plaintexts and ciphertexts. In other words, the following arithmetic holds true:

$$
\operatorname{Dec}\left(\operatorname{Enc}\left(m_{1}\right)+\operatorname{Enc}\left(m_{2}\right) \bmod q\right)=m_{1}+m_{2},
$$

as long as $\left|m_{1}+m_{2}\right|<p / 2$.

Regarding the multiplication $m_{1} \times m_{2}$ over encrypted data, we let the multiplicand $m_{2} \in \mathbb{Z}_{p}$ be encrypted with Enc, and use a different encryption process for the multiplier $m_{1} \in \mathbb{Z}_{p}$, denoted by Enc2, defined as

$$
\operatorname{Enc} 2\left(m_{1}\right)=m_{1} R+\mathbf{O}=: \mathbf{M}_{\mathbf{1}},
$$

where $\mathbf{O}=\operatorname{Enc}\left(0_{\log (q)-(N+1) \times 1}\right)$ is an encrypted zero vector, and $R$ is defined as:

$$
R:=\left[10^{0}, 10^{1}, 10^{2}, \cdots, 10^{\log (q)-1}\right]^{T} \otimes I_{N+1},
$$

with $\otimes$ being the Kronecker product.

Then, as any ciphertext $c$ in $\mathbb{Z}_{q}^{1 \times(N+1)}$ can be represented as $c=\sum_{i=0}^{\log (q)-1} 10^{i} c_{i}$, where $c_{i}$ 's components are single digits from 0 to 9 , a decomposition function $D$ can be used to decompose the ciphertext by its digits in the form

$$
D(c):=\left[c_{0}, c_{1}, \cdots, c_{\log (q-1)}\right] .
$$

Since $c=D(c) R$, the multiplication of ciphertexts can be computed as

$$
\mathbf{M}_{\mathbf{1}} \times_{\mathcal{C}} \operatorname{Enc}\left(m_{2}\right):=D\left(\mathbf{m}_{\mathbf{2}}\right) \cdot \mathbf{M}_{\mathbf{1}} \bmod q
$$

\footnotetext{
${ }^{1}$ For the error distribution, we assume that the parameter $L$ is chosen sufficiently large and neglect the probability that $|e| \geq r / 2$, with some $r<L$.
} 
Consequently, we have the following property of homomorphic multiplication using both Enc and Enc2

$$
\operatorname{Dec}\left(\operatorname{Enc} 2\left(m_{1}\right) \times_{\mathcal{C}} \operatorname{Enc}\left(m_{2}\right)\right)=m_{1} m_{2},
$$

as long as $\left|m_{1} m_{2}\right|<p / 2$.

Instead of using two encryption processes as above for multiplication operation, one can also consider the use of LWE-based cryptosystem in (1) and (8) to perform multiplication of a ciphertext by a plaintext, where multiplication is considered as repeated homomorphic addition. However, such an approach is not recommended as it compromises the security and privacy of the closed-loop systems by potential leakage of information from the interception of a plaintext multiplier.

\section{B. Infinite Time Horizon Encryption}

When we implement the aforementioned ciphertext operations repeatedly in a closed-loop control system with a dynamic controller in the feedback loop, the significand of the encrypted state will tend to accumulate. A notable exception to this problem is the class of linear dynamic system whose state matrix consists of integers, as observed in [12]. Let us consider the following discrete-time controllers

$$
\left\{\begin{aligned}
x(t+1) & =F x(t)+G y(t), \\
u(t) & =H x(t),
\end{aligned}\right.
$$

where, $t \in \mathbb{Z}_{+}, x(t)$ is the controller state variables with the initial values $x_{0}, y(t)$ is the plant's output, $u(t)$ is the controller's output and $F, G, H$ are system's matrices with $F$ defined by integers. For the application of $\mathrm{HE}$ in the feedback loop, the plant's output $y(t)$ is converted to integers by choosing an arbitrary scaling parameter $s_{2} \geq 1$ so that

$$
\overline{\bar{y}}(t):=\left\lceil\frac{y(t)}{s_{2}}\right\rfloor
$$

corresponds to the plant's output in integer form. Then, the system is converted into a system over integers by rounding $G$ using a scaling factor $1 / s_{1} \geq 1$, such that

$$
\left\{\begin{array}{l}
\overline{\bar{x}}(t+1)=F \overline{\bar{x}}(t)+\left\lceil\frac{G}{s_{1}}\right\rfloor \overline{\bar{y}}(t), \\
\overline{\bar{u}}(t)=\left\lceil\frac{H}{s_{3}}\right\rfloor \overline{\bar{x}}(t), \\
\overline{\bar{x}}(0)=\left\lceil\frac{x_{0}}{s_{1} s_{2}}\right\rfloor,
\end{array}\right.
$$

where $1 / s_{3} \geq 1$ is the scale factor for the matrix $H$. Finally, the systems' matrices are encrypted as multipliers as

$$
\mathbf{F}=\operatorname{Enc} 2(F), \mathbf{G}=\operatorname{Enc} 2\left(\left\lceil\frac{G}{s_{1}}\right\rfloor\right), \mathbf{H}=\operatorname{Enc} 2\left(\left\lceil\frac{H}{s_{3}}\right\rfloor\right) .
$$

With the encrypted matrices the dynamic controller becomes

$$
\left\{\begin{array}{l}
\mathbf{x}(t+1)=\mathbf{F} \times_{\mathcal{C}} \mathbf{x}(t)+\mathbf{G} \times_{\mathcal{C}} \mathbf{y}(t), \\
\mathbf{u}(t)=\mathbf{H} \times_{\mathcal{C}} \mathbf{x}(t), \\
\mathbf{x}(0)=\operatorname{Enc}\left(\left\lceil\frac{x_{0}}{s_{1} s_{2}}\right\rfloor\right),
\end{array}\right.
$$

where $\mathbf{x}(t)$ and $\mathbf{u}(t)$ is the encrypted state and output of the controller, respectively, and $\mathbf{y}(t)$ is the encrypted plant output, defined as $\mathbf{y}(t)=\operatorname{Enc}(\overline{\bar{y}}(t))$. In case of the first-order system, the output matrix $H$ can be set as $H=1$, without loss of generality. Then, the encryption of $H$ as well as the multiplication $\times_{\mathcal{C}}$ for the output $\mathbf{u}(t)$ becomes dispensable, and we can let $\mathbf{u}(t)=\mathbf{x}(t)$.

Regarding the performance error of the encrypted controller, it is attributable to two factors; error due to quantization (round operation for matrices and signals) and injection of errors during encryption. We note that the effect of both of them can be arbitrarily small, by appropriate choice of the scale factors $\left\{s_{1}, s_{2}, s_{3}\right\}$, and the parameters of the cryptosystem. See [11], for more details.

\section{Distance-based Distributed Formation Control}

For the distributed formation control, we consider the use of well-known distance-based gradient control with estimators as presented in [15]. We consider a formation of $n$ mobile robots that move on a plane, e.g., they operates in a 2-dimensional space.

The formation of mobile robots/agents can be described using formation graph $\mathcal{G}=(\mathcal{V}, \mathcal{E})$, where $\mathcal{V}$ is the set of vertices, each vertex $v \in \mathcal{V}$ represents an agent, and the set of edges $\mathcal{E} \subseteq \mathcal{V} \times \mathcal{V}$ contains pairs of agents that have to maintain prescribed distance of a given formation shape. We assume that $\mathcal{G}$ is undirected. The set $N_{i}$ denotes the set of neighbours of agent $i$ given by $N_{i}:=\{j \in \mathcal{V}:(i, j) \in \mathcal{E}\}$. For the undirected graph $\mathcal{G}$, we can define the incidence matrix $B=\left\{b_{i k}\right\} \in \mathbb{R}^{|\mathcal{V}| \times|\mathcal{E}|}$ by assigning arbitrarily directionality in the graph as follows

$$
b_{i k} \triangleq\left\{\begin{aligned}
+1 & \text { if } i=\mathcal{E}_{k}^{\text {tail }} \\
-1 & \text { if } i=\mathcal{E}_{k}^{\text {head }} \\
0 & \text { otherwise }
\end{aligned}\right.
$$

where $\mathcal{E}_{k}^{\text {tail }}$ and $\mathcal{E}_{k}^{\text {head }}$ denote the tail and head nodes of edge $\mathcal{E}_{k}$ respectively. Using the formation graph $\mathcal{G}$, one can deploy the well-studied gradient-based distributed formation control law where each agent maintains the desired distance with its neighbors in order to form an infinitesimally rigid formation shape [14]. In particular, each robot uses local measurement systems to obtain relative position with respect to the local coordinate system.

Using $\mathcal{G}$ and the agent positions $p=\left[\begin{array}{llll}p_{1} & p_{2} & \ldots & p_{n}\end{array}\right]$, we can define formation shape as follows. Let $\bar{B}=B \otimes$ $I_{2}$. The measured relative positions defined according to the edge $\mathcal{E}$ can now be described by

$$
z=\bar{B}^{T} p
$$

where each component $z_{k}=p_{i}-p_{j}$ in $z$ corresponds to the relative position associated with the edge $\mathcal{E}_{k}=(i, j)$. For a given admissible target shape, we can define a vector of desired inter-agent distances $d^{*} \in \mathbb{R}^{|\mathcal{E}|}$ defined on $\mathcal{E}$. The set of all desired equilibrium points is then given by

$$
\mathcal{D}:=\left\{p \in \mathbb{R}^{2 n}:\left\|z_{k}\right\|=d_{k}, k \in\{1, \ldots, \mathcal{E}\}\right\} .
$$

For detailed analysis on this formation graph, on the characterization of infinitesimally rigid formation and on the 
standard gradient-based distributed formation control, we refer interested readers to [13], [15], [17].

Assuming that every agent is described by a kinematic point and evolves according to a single integrator, the standard distance-based gradient control law for maintaining the formation is given by

$$
u=-c_{1} \bar{B} D_{z} D_{\tilde{z}} e,
$$

where $\tilde{z}$ is the stacked vector of $\left\|z_{k}\right\|^{-1}, c_{1}$ is a constant gain, $e=\operatorname{col}_{k}\left\{\left\|z_{k}\right\|-d_{k}^{*}\right\}$ is the column vector of the distance error at every edge with $d_{k}^{*}$ as the desired distance for the $k$-th edge, $D_{z}$ and $D_{\bar{z}}$ represent the block diagonal matrix of $z$ and $\bar{z}$, respectively. When there is no discrepancy on the distance constraint between pairing agents in all edges and no measurement noise, the control law (17) ensures local exponential stability to the equilibrium set $\mathcal{D}$, e.g., the desired formation shape is attained. However, this nice stability property is destroyed when there is a discrepancy/disagreement of the desired distance by agents in an edge. It can happen, for instance, when there is a constant bias in the local range sensor systems. In this paper, we will adopt the solution that is proposed in [14] where a distributed dynamic estimator is deployed to one of the agents in every edge. Particularly, we will adopt the computation of the distributed dynamic estimator in the cloud that is secured by encryption.

\section{Distributed Dynamic Estimators for Mismatch Compen- sation}

As given in the Introduction, the presence of measurement biases or mismatches $\mu \in \mathbb{R}^{|\mathcal{E}|}$ in the distance constraint can induce undesired formation shape and result in group motion as studied in [16]. In these circumstances, de Marina et al. [14] have proposed distributed estimators that can be deployed at a vertex of every edges to robustly compensate these mismatches. For each edge $\mathcal{E}$, a local estimator is defined to the agent $\mathcal{E}_{k}^{\text {tail }}$ (called an estimating agent) and is given by

$$
\begin{aligned}
\dot{\xi}_{k} & =\kappa\left(e_{k}^{\text {tail }}-\hat{\mu}_{k}\right) \\
\hat{\mu}_{k} & =\xi_{k},
\end{aligned}
$$

where the estimator state $\hat{\mu}_{k}$ aims to compensate for the unknown mismatch or measurement bias $\mu_{k}$ with respect to the agent $\mathcal{E}_{k}^{\text {head }}$, and $\kappa>0$ is the estimator gain. Note that $e_{k}^{\text {tail }}$ and $e_{k}^{\text {head }}$ is related by $e_{k}^{\text {tail }}-\mu_{k}=e_{k}^{\text {head }}$. Thus when the estimator state $\hat{\mu}_{k}(t) \rightarrow \mu_{k}$ as $t \rightarrow \infty$, the formation will be defined by $e_{k}^{\text {head }}$ and the shape will be based on the prescribed distance in $e_{k}^{\text {head }}$. Using (17) and (18), the combined estimator and gradient-based formation control law is given by

$$
u=-c_{1} \bar{B} D_{z} D_{\tilde{z}} e-c_{2} \bar{B}^{\text {est }} D_{\tilde{z}} \hat{\mu},
$$

where $c_{2}$ is a constant gain, $\hat{\mu} \in \mathbb{R}^{|\mathcal{E}|}$ is the stacked column vector of $\hat{\mu}_{k}$ and $\bar{B}^{\text {est }}=B^{\text {est }} \otimes I_{2}$ with $B^{\text {est }}=\left\{b_{i k}\right\} \in$ $\mathbb{R}^{|\mathcal{V}| \times|\mathcal{E}|}$ being a matrix whose elements is given by

$$
b_{i k} \triangleq\left\{\begin{array}{l}
1 \text { if } i=\mathcal{E}_{k}^{\text {tail }} \\
0 \text { if } i=\mathcal{E}_{k}^{\text {head }} \\
0 \text { otherwise. }
\end{array}\right.
$$

Note that the matrix $\bar{B}^{\text {est }}$ is an indicator matrix for the estimating agent in every edge.

\section{ROBUST DISTRIBUTED FORMATION CONTROL WITH ENCRYPTION}

In this section, we present the use of an edge/cloud computer to realize the aforementioned distributed formation control with estimator, where the local sensor measurement and distance constraint remain private to the individual agent. In this case, the edge/cloud computer does not act as a centralized computer and is agnostic on the specific knowledge of the agents. The edge computer will compute both the evolution of individual state estimator and the resulting control input in ciphertext. The resulting individual control input is then returned to each agent in ciphertext that can be decrypted using the individual key known only to each agent. This study demonstrates the applicability of HE with infinite-time horizon implementation for the control of multi-agent systems that collaboratively execute a task (maintaining a formation shape, in this case) using only their local information and without sharing information to the neighbours via public edge/cloud computing infrastructure.

\section{A. Scaled Logarithmic Quantization for Scaling Data}

In the application of HE, one crucial element to communicate real-time data between the agent and the edge/cloud computer is the use of scaled quantization. Given a realtime value of a variable $\nu_{k} \in \mathbb{R}$, which is available to an agent in the $k$-th edge (e.g., the distance $d_{k}$, the elements in relative position vector $z_{k}$, etc), it will be scaled (up or down) and rounded so that it can be encrypted based on the allocated plaintext space. As commonly done, we can perform the scaled quantization to the variable $\nu_{k}$ by $\overline{\bar{\nu}}_{k}=\left\lceil S_{\nu_{k}} \nu_{k}\right\rfloor \in \mathbb{Z}$ where the scaling factor $S_{\nu_{k}}>1$ must be chosen appropriately. The same scaling factor will be used again to rescale the decrypted control input from the edge/cloud computer.

In general, there are two issues in the use of static scaling factor $S_{\nu_{k}}$. Firstly, the scaled quantization is known to introduce scaled quantization error that will affect the stability of the closed-loop system. In particular, when there is a memory element in the feedback loop, the memory state can accummulate such quantization error that can deteriorate the closed-loop systems performance and hence it requires regular resetting of controller state. Secondly, as the use of static scaling factor is the same as using uniform quantizer, we can only guarantee practical stability where steady-state error will occur due to the rounding precision. We refer to [18] on general practical stability analysis for passive systems using such uniform quantization, which represents also the mobile robot dynamics with gradient-based control law.

Inspired by the use of logarithmic quantizer, which can guarantee asymptotic stability of closed-loop systems [19], we consider in this work the use of scaled logarithmic quantizer where the scaling factor $S_{\nu_{k}}$ changes in a logarithmic fashion. Intuitively, we want to maximize $S_{\nu_{k}}$ to 
ensure that we maintain the precision and stability of the system. However, as defined in Section II-A the upper-bound condition on the message $|m|<p / 2$ limits our ability to scale the data arbitrarily.
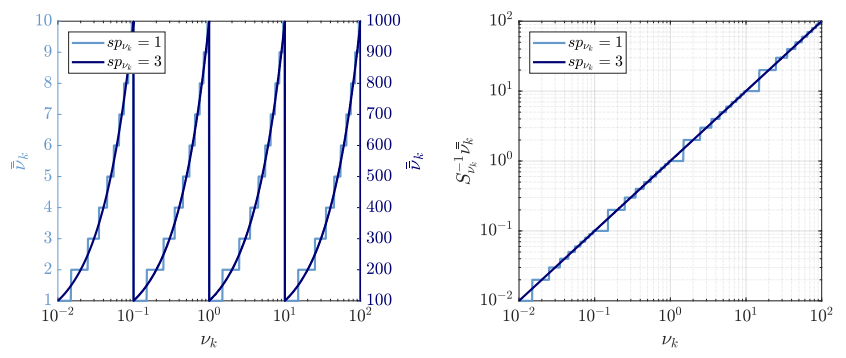

Fig. 2. Left: The plot of $\overline{\bar{\nu}}_{k}=\left\lceil S_{\nu_{k}} \nu_{k}\right\rfloor$ with $S_{\nu_{k}}$ be as in 21 and with $s p_{\nu_{k}}=1$ (in light blue) and $s p_{\nu_{k}}=3$ (in dark blue). The abscissa is $\nu_{k}$ in log-scale and ordinate is $\overline{\bar{\nu}}_{k}$; Right: The plot of $S_{\nu_{k}}^{-1} \overline{\bar{\nu}}_{k}$ in log-scale with $s p_{\nu_{k}}=1$ (in light blue) and $s p_{\nu_{k}}=3$ (in dark blue).

The logarithmic design of $S_{\nu_{k}}$ is based on prioritizing the significant figure of $\nu_{k}$ than its decimal values. Accordingly, for each variable of interest $\nu_{k}$, we define a desired significant figures $s p_{\nu_{k}}$ and the scaling factor is given by

$$
S_{\nu_{k}}=10^{s p_{\nu_{k}}-\left\lfloor\log _{10}\left(\left|\nu_{k}\right|\right)\right\rfloor-1} \text {. }
$$

This allows us to send the information in the data up to a prescribed significant number.

Using the logarithmic scaling factor as above, we have that changes in the magnitude of the measures as the agents move closer or further apart do not affect the maximum magnitude of the information sent. Figure 2 shows the plot of the scaled logarithmic quantization for different value of $s p_{\nu_{k}}$. It shows that the scaled logarithmic quantizer can approximate an identity operator well in contrast to the use of uniform quantizer that becomes zero below a certain threshold value. As illustrated in this figure as well, the choice of significant figure $s p_{\nu_{k}}$ may cause the message to be larger than the allowable plaintext space, i.e., $\left|\overline{\bar{\nu}}_{k}\right|>p / 2$. Therefore, we will now define the next step to determine the bounds for the plaintext space $p$. Note that the minimum required plaintext space can be calculated based on the desired significant figures and amount of ciphertext operations to be carried out. Based on a number of multiplied variables $m$ with different $s p_{i}, i \in\{1, \ldots, m\}$, and based on the number of added variables $n$ with different $s p_{j}, j \in\{1, \ldots, n\}$, we can have the following conservative lower bound of $p$

$$
p>\prod_{i=0}^{m} 10^{2 s p_{i}}+2 \sum_{j=0}^{n} 10^{s p_{j}} .
$$

On the other hand, the upper bound of $p$ can generally be determined by the precision of the data format used to perform the calculations. A 64-bit signed integer would require $p<2^{63}-1$. Although this maximum is enough to contain the desired messages, there is a caveat of defining a large $p$ in the application process. That is, the magnitude of $p$ also defines the upper boundary $L p$ of the elements of a ciphertext. Therefore, the resulting values of one multiplication operation will have a maximum of $(L p)^{2}$. Using signed integers this quickly results in data overflow. Hence,

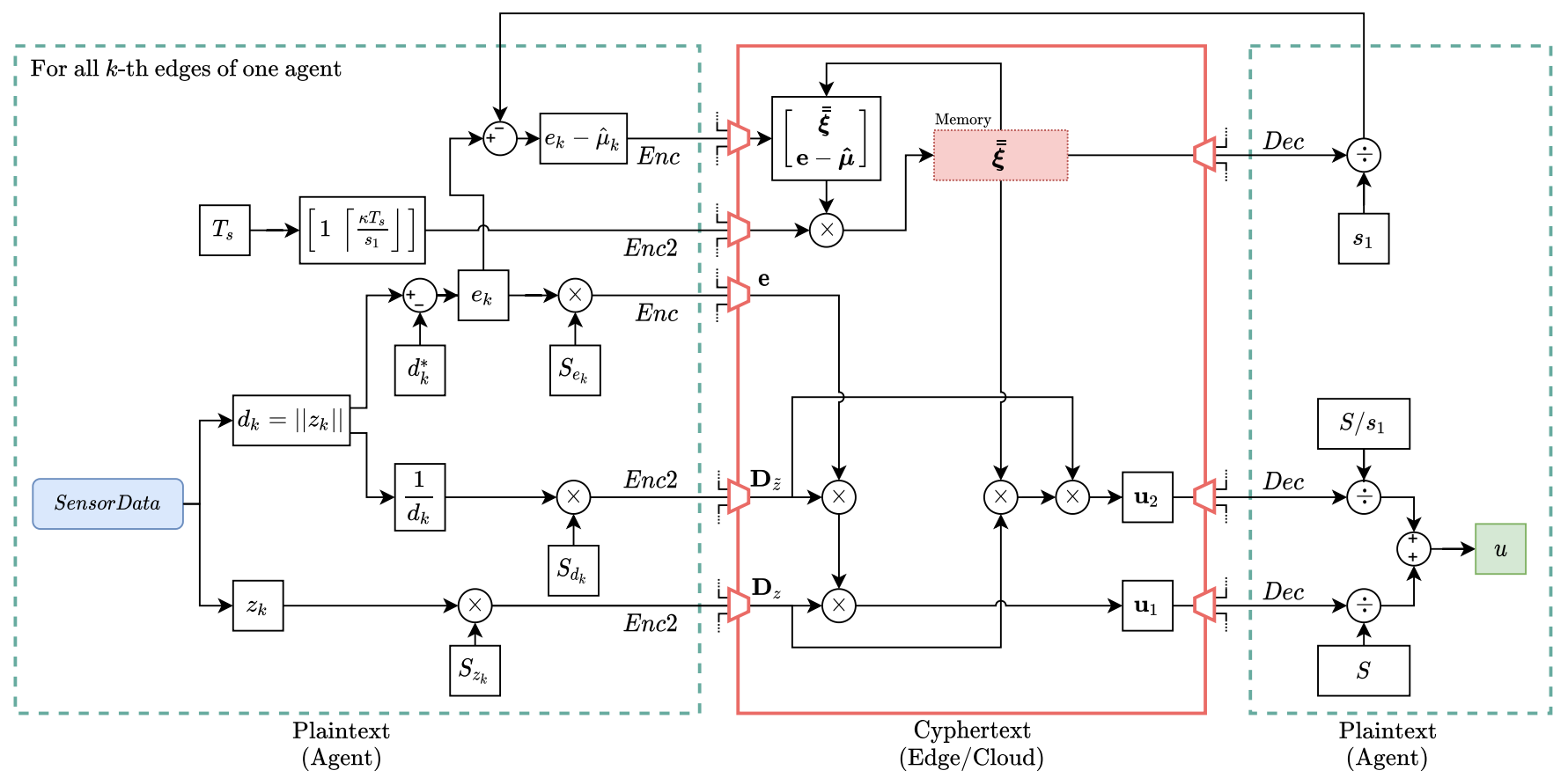

Fig. 1. General data flowchart of HE-enabled distributed formation control with estimator which are deployed in the edge/cloud. All variables are described in Section II.C, II.D, III.A and III.B. The isosceles trapezoids represent multiplexers and demultiplexers that combine and split data from and to all edges, respectively. For brevity, the operations in the edge/cloud uses the concatenated data while in practice, they can be computed "locally" in the edge/cloud per agent. The input data to the flowchart is the on-board sensor measurement and the output data is the control input $u$ to be implemented in each robotic agent. 
at the expense of memory and performance, we avoid this limit by making use of an arbitrary-precision integer which is provided, for instance, by the programming language Python.

\section{B. Encrypting Estimator Dynamics}

As we implement the distributed estimators with its own state variable in the edge/cloud, we encrypt them as follows. First we consider the following discrete-time version of (18)

$$
\begin{aligned}
\xi(t+1) & =\xi(t)+T_{s} \kappa\left(e^{\text {tail }}(t)-\hat{\mu}(t)\right) \\
\hat{\mu}(t) & =\xi_{k}(t)
\end{aligned}
$$

where $t \in \mathbb{Z}_{+}$is the discrete-time, and $T_{s}>0$ is the sampling time. Correspondingly, with a scale factor $1 / s_{1} \geq$ 1 , we convert the system 23 as

$$
\overline{\bar{\xi}}_{k}(t+1)=\overline{\bar{\xi}}_{k}(t)+\left\lceil\frac{T_{s} \kappa}{s_{1}}\right\rfloor\left(e_{k}^{\text {tail }}(t)-\hat{\mu}_{k}(t)\right),
$$

in which, the error $\left\|\xi_{k}(t)-s_{1} \overline{\bar{\xi}}_{k}(t)\right\|$ tends to zero as $s_{1}$ tends to zero, so that the output $\hat{\mu}_{k}(t)$ can be obtained by $\hat{\mu}_{k}(t)=$ $s_{1} \overline{\bar{\xi}}_{k}(t)$. As the equation 24 uses only integer coefficients, it can directly be implemented in the edge/cloud environment using HE while the data of $e_{k}^{\text {tail }}(t)-\hat{\mu}_{k}(t)$ is transmitted by the agent as integers via standard scaled quantizer. Hence, the encrypted estimator state variable will evolve as an integer in the ciphertext and, when it is returned back to the agent, its value is decrypted and scaled back by the same scaling constant $s_{1}$.

As the estimator state always evolves in the ciphertext, it can be implemented with an infinite time horizon. On the other hand, it has been shown in [14] that the closed-loop system (without encryption) is locally exponentially stable, so that it is also locally input-to-state stable. Therefore, the error due to the use of scaled logarithmic quantizer will lead to practical stability, e.g., steady-state error can occur which is close to zero as a logarithmic scale is used.

In order to secure the privacy of the coefficients of the estimators, we apply encryption Enc2 to the coefficients 1 and $\left\lceil\frac{T_{s} \kappa}{s_{1}}\right\rfloor$ which can be computed offline and stored in the edge/cloud.

\section{Dataflow Diagram}

Based on the description in the previous subsections, we can now present the architecture of the HE-enabled formation control with mismatch estimator. Figure 1 shows the data flowchart from the edges of an agent to the edge/cloud where the distributed formation control computation is performed and returns the formation control input according to (19) implemented in the ciphertext. As shown in this figure, the scaled logarithmic quantization is applied to the distancebased formation control part (e.g., (17)), while the estimator part (the last term in (19) and (18)) uses standard scaled quantization with fixed $S_{\nu_{k}}$ prior to the encryption. Here, the decrypted $\hat{\mu}(t+1)$ is used to compute $e_{k}(t+1)-\hat{\mu}(t+1)$ for the next sampling time.

\section{Simulation Results}

\section{A. Simulation Setup}

In this section, we show and evaluate the performance and robustness of the proposed HE-enabled distance-based formation control with estimators. For simplicity, we consider the formation of three agents forming a triangle. Figure 3 shows the formation graph where estimating agents are shown at the tail of each edge.

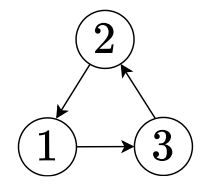

Fig. 3. The formation graph used in simulations where the three agents are represented by the three nodes. The direction of the graph indicates the estimating agent for each edge, i.e. the node at the tail of each arrow.

All simulations were carried out on a laptop running ROS [20] Melodic in Ubuntu 18.04 with an i7-4700MQ CPU, GT 740M GPU and 8GB of RAM. For the simulation setup in the Gazebo environment, we use LIDAR as the onboard distance sensor in each robot. The ROS robot model is based on the Nexus ${ }^{\circledR}$ mobile robot equipped with four $100 \mathrm{~mm}$ Mecanum ${ }^{\circledR}$ wheels. The control input will be the longitudinal and lateral velocity of the robot. For all agents, the desired distances for the distance-based formation control are set to $d_{k}^{*}=0.8$, with a sampling time $T_{s}=0.1[\mathrm{~s}]$, and initial estimator state $\overline{\bar{\xi}}_{k}=0$. Unless stated otherwise, the encryption within the simulations was carried out with a key length of $N=10$, an injected error of $e=100$, and an available plaintext space of $p=10^{13}$.

\section{B. Simulation Result}

For comparing the performance of the closed-loop systems with and without encryption with infinite-time horizon, we performed a simulation of the closed-loop system for both scenarios where agent 1 has an initial distance error of $e_{k}=0.1$ and the same distance mismatch of $\mu_{k}=0.1$ with respect to the other two agents. Figure 4 shows the performance of the HE-enabled formation control is close to that of the original one. In particular, the encrypted estimator states are able to converge to the correct ones with similar convergence rate as the original one. More importantly, in this simulation, the evolution of estimator states remains encrypted throughout simulation time without any problem.

\section{Robustness Analysis}

One important aspect to the security of a homomorphically encrypted system is the role of key length $N$. In this regard, we perform robustness analysis of the formation convergence via Monte Carlo analysis for multiple values of $N$. For brevity, we only present the convergence of one agent's distance to one of its neighbours. The similar behaviour is also exhibited by the other agents.

We consider four different values of $N=30,60,90$ and 120 and for each value of $N$, we run fifty simulations with initial distance of $d_{k}=1$ and constant mismatch of 


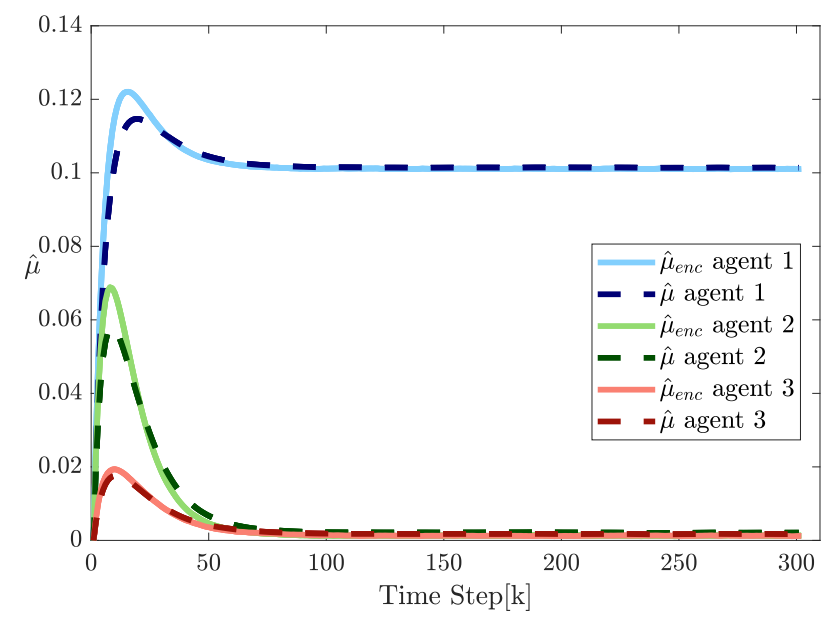

Fig. 4. Simulation result of the closed-loop system with and without encryption in the formation control. The plot shows the settling performance of $\hat{\mu}_{\text {enc }}$ (encrypted) against $\hat{\mu}$ (non-encrypted) where agent 1 is assigned a mismatch of $\mu_{k}=0.1$ with respect to its neighbor.

$\mu_{k}=0.1$ in agent 1 . In this simulation, the source of uncertainties is on the computational resources for the encryption and for control computation that introduce overhead and asynchronous simulation time in each simulated robot. Figure 5 shows the transient behaviour for all $N$ with $95 \%$ confidence interval indicating the aforementioned variability between simulations. This simulation shows degradation in the convergence of formation for larger values of $N$, which is mainly due to the update time of the agent's velocity caused by the encryption. In Figure 6 , we plot the measured run-time of the executed operations on one integer for increasing $N$ and we can see that the increase in key length dramatically affects the time taken to encrypt a value using Enc2, which directly impacts the time between control inputs to an agent.

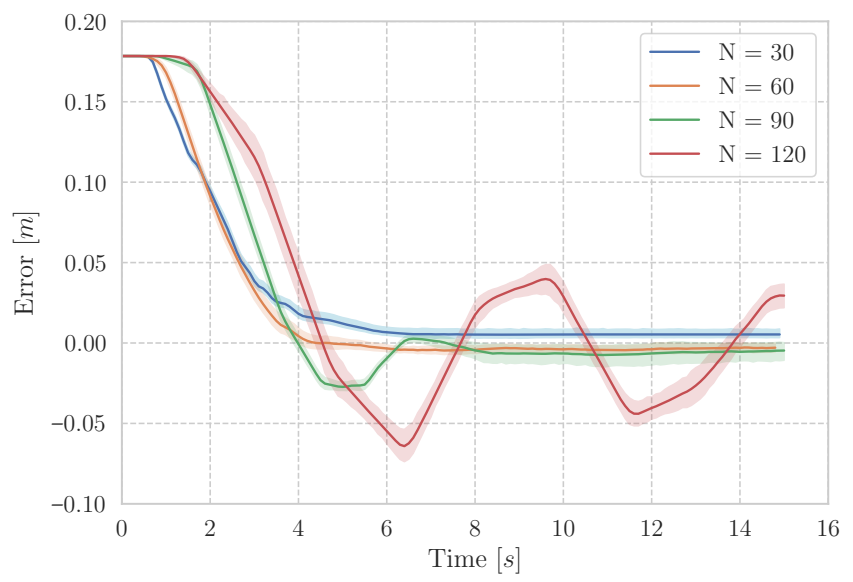

Fig. 5. Effect of key length $N$ on an agent's transient behaviour of the error in distance with respect to a neighbour. A $95 \%$ confidence interval indicates the distribution of convergence over 50 simulations per value of $N$.

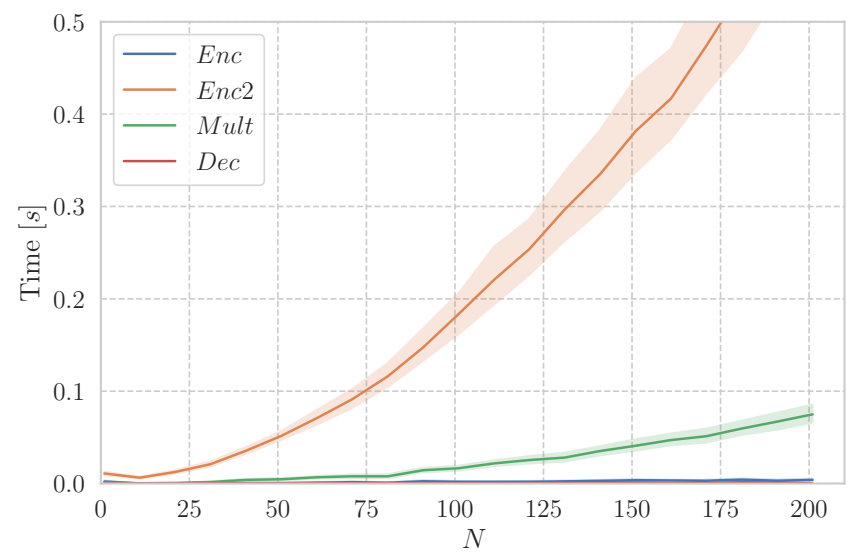

Fig. 6. Effect of $N$ on run-time execution of encryption (Enc, Enc2) and decryption $(D e c)$ of an integer, as well as multiplication (Mult) of two encrypted values. A 95\% confidence interval indicates the distribution of run-time over 50 simulations per operation type.

\section{CONCLUSIONS AND Future WORKS}

In this work, we presented an HE-enabled distributed formation control with estimators. We proposed the use of a scaled logarithmic quantizer in combination with the transformation of the estimator to operate over integers. This allows us to secure the privacy of the controller in the edge/cloud computer where the estimator states evolve completely in ciphertext (i.e., it is always encrypted) with infinitetime horizon. Simulation results are presented that show the efficacy and robustness of the proposed architecture.

There are some limitations that affect the design of the encrypted controller and the simulations that should be considered in future approaches. The limited computational power has affected the convergence time when larger $N$ (improved security) is used. One can deploy high-computing infrastructure to ensure that the encryption process does not become the bottleneck in the edge/cloud so that the convergence time is not affected.

The use of single integrator as the estimator is to compensate for constant mismatches in the distance constraints. When there are (periodic) disturbance/reference signals that are generated by known exosystems, we can also deploy dynamic internal models in the edge/cloud.

In order to compute the large integers generated in the encryption process we made use of arbitrary precision arithmetics offered natively in Python. Also termed "multiple precision", this challenge is reoccurring in the implementation of cryptography [21]. Although less efficient than using fixed-precision data types, we found it fast enough to perform the needed operations for this proof of concept. Further research is necessary to analyse the concrete impact on the encryption time, and settling of the formation.

Finally, we provide some remarks in dealing with the computational complexity in HE-enabled distributed control systems:

- Encryption of static parameters off-line. The encryption of the matrix parameters as static multipliers can be performed off-line, during initialization. Thus, despite the 
applied encryption algorithm for the matrices requiring a relative large amount of computational resources, it would not be a burden for real-time control operation.

- The use of non-private data as unencrypted. For example, by making public the use of the integral controller in the proposed method, the associated state matrices that do not contain any private information can be kept unencrypted.

\section{ACKNOWLEDGEMENT}

We would like to thank prof. Hyungbo Shim from Seoul National University for the initial discussion and problem formulation setup.

\section{REFERENCES}

[1] N. Aleisa, "A comparison of the 3des and aes encryption standards," International Journal of Security and Its Applications, vol. 9, no. 7, pp. 241-246, 2015.

[2] Y. Yang, L. Wu, G. Yin, L. Li, and H. Zhao, "A survey on security and privacy issues in internet-of-things," IEEE Internet of Things Journal, vol. 4 , no. 5 , pp. $1250-1258,2017$.

[3] H. Sandberg, S. Amin, and K. H. Johansson, "Cyberphysical security in networked control systems: An introduction to the issue," IEEE Control Systems Magazine, vol. 35, no. 1, pp. 20-23, 2015.

[4] P. Parmar, S. Padhar, S. Patel, N. Bhatt, and R. Jhaveri, "Survey of various homomorphic encryption algorithms and schemes," International Journal of Computer Applications, vol. 91, 032014.

[5] A. B. Alexandru and G. J. Pappas, "Secure multi-party computation for cloud-based control," in Privacy in Dynamical Systems, F. Farokhi, Ed. Singapore: Springer, 2020, pp. 179-208.

[6] J. Kim, H. Shim, and K. Han, "Comprehensive introduction to fully homomorphic encryption for dynamic feedback controller via lwebased cryptosystem," in Privacy in Dynamical Systems, F. Farokhi, Ed. Singapore: Springer, 2020, pp. 209-230.

[7] M. S. Darup, "Encrypted model predictive control in the cloud," in Privacy in Dynamical Systems, F. Farokhi, Ed. Singapore: Springer, 2020, pp. 231-266.
[8] K. Kogiso, "Encrypted control using multiplicative homomorphic encryption," in Privacy in Dynamical Systems, F. Farokhi, Ed. Singapore: Springer, 2020, pp. 267-286.

[9] C. Murguia, F. Farokhi, and I. Shames, "Secure and private implementation of dynamic controllers using semi-homomorphic encryption," IEEE Transactions on Automatic Control, vol. PP, pp. 1-1, 052020.

[10] J. Kim, C. Lee, H. Shim, J. Cheon, A. Kim, M. Kim, and Y. Song, "Encrypting controller using fully homomorphic encryption for security of cyber-physical systems," IFAC-PapersOnLine, vol. 49, pp. 175-180, 122016

[11] J. Kim, H. Shim, and K. Han, "Dynamic controller that operates over homomorphically encrypted data for infinite time horizon," ArXiv, vol. abs/1912.07362, 2019.

[12] J. H. Cheon, K. Han, H. Kim, J. Kim, and H. Shim, "Need for controllers having integer coefficients in homomorphically encrypted dynamic system," in 2018 IEEE Conference on Decision and Control (CDC), 2018, pp. 5020-5025.

[13] K.-K. Oh, M.-C. Park, and H.-S. Ahn, "A survey of multi-agent formation control," Automatica, vol. 53, pp. 424-440, 2015.

[14] H. G. De Marina, M. Cao, and B. Jayawardhana, "Controlling rigid formations of mobile agents under inconsistent measurements," IEEE Transactions on Robotics, vol. 31, no. 1, pp. 31-39, 2015.

[15] H. G. De Marina, B. Jayawardhana, and M. Cao, "Distributed rotational and translational maneuvering of rigid formations and their applications," IEEE Transactions on Robotics, vol. 32, no. 3, pp. 684697, 2016.

[16] S. Mou, M.-A. Belabbas, A. Morse, Z. Sun, and B. Anderson, "Undirected rigid formations are problematic," IEEE Transactions on Automatic Control, vol. 61, no. 10, pp. 2821-2836, 2016.

[17] B. D. O. Anderson, C. Yu, B. Fidan, and J. Hendrickx, "Rigid graph control architectures for autonomous formations," IEEE Control Systems Magazine, vol. 28, pp. 48-63, 2008.

[18] C. De Persis and B. Jayawardhana, "Coordination of passive systems under quantized measurements," SIAM Journal on Control and Optimization, vol. 50, no. 6, pp. 3155-3177, 2012.

[19] M. Fu and L. Xie, "The sector bound approach to quantized feedback control," IEEE Transactions on Automatic Control, vol. 50, no. 11, pp. 1698-1711, 2005.

[20] M. Quigley, K. Conley, B. P. Gerkey, J. Faust, T. Foote, J. Leibs, R. Wheeler, and A. Y. Ng, "Ros: an open-source robot operating system," in ICRA Workshop on Open Source Software, 2009.

[21] T. St Denis, BigNum Math: implementing cryptographic multiple precision arithmetic. Elsevier, 2006. 